\title{
Precision Medicine for Metastatic Breast Cancer
}

\author{
Elise Deluche, MD, Elisa Onesti, MD, and Fabrice Andre, MD
}

\section{OVERVIEW}

Genomic studies have shown that large numbers of candidate targets are observed in breast cancer. Nevertheless, only a few of them are validated as relevant targets in clinical studies. Estrogen receptor (ER) and HER2 expressions could be associated with a level I evidence. Beyond ER and HER2, BRCA and PIK3CA mutations (when targeted with alpha-specific PI3K inhibitors) could be considered as promising targets in breast cancer since they have been associated with objective responses in phase $\mathrm{I} / \mathrm{II}$ trials. In addition to these four molecular alterations, several others have shown promising results in preclinical studies and are being investigated in clinical trials. These genomic alterations include AKT1, ERBB2, and ESR1 mutations. These considerations highlight the lack of evidence for using multiplex technologies to individualize therapy in metastatic breast cancer. Sequencing multiple genes to treat metastatic breast cancer is very promising but should be done in the context of clinical trials, either to enrich phase $1 / I$ trials in patients with genomic alterations or to show medical usefulness of new biotechnologies like next-generation sequencing (NGS). Although most current approaches of precision medicine are aiming at targeting drivers, additional applications could be developed in the future. This includes the identification of DNA repair deficiencies, mechanisms of immune suppression, and identification of minority lethal subclones. Finally, one of the very promising applications of genomics for metastatic breast cancer is the identification of pathway activation or defects at the individual level. For example, gene expression and single nucleotide polymorphisms (SNP) signatures are being developed to detect kinase (such as mammalian target of rapamycin [mTOR]/CDK4) activations or DNA repair deficiencies.

$\mathrm{M}$ olecular studies have shown that breast cancer includes a large number of subgroups defined by the presence of a specific genomic or protein alteration. ER expression was the first validated target in breast cancer, leading to the optimal development of endocrine therapy. ${ }^{1}$ In the late 1990s, HER2 overexpression was validated as a target and was shown to be a predictive biomarker for the efficacy of trastuzumab. ${ }^{2}$ During the 2000s, genomic analyses based on gene expression arrays suggested that breast cancer could be divided into four different subgroups: luminal A, luminal B, HER2-enriched, and basal-like. ${ }^{3-5}$ Further studies have suggested that basal-like cancers can be subdivided into six subgroups. ${ }^{6}$ More recently, studies on NGS have suggested that approximately 40 genomic alterations can be found in primary breast cancers. ${ }^{7,8}$ Overall, this introduction emphasizes that each single breast cancer presents a specific molecular profile and specific molecular mechanisms of cancer progression. Parallel to the advances in the understanding of disease biology, several advances in technology could dramatically change patient care. Indeed, it has been shown that highthroughput DNA sequencing together with comparative genomic hybridization (CGH; copy number analyses) can be performed robustly in the clinical practice. ${ }^{9,10}$ This has led to the development of precision medicine that involves multiplex molecular analyses to identify molecular mechanisms of cancer progression in each individual in order to improve treatment. In the following review, we present the current state of this approach in metastatic breast cancers (mBC). Several technologies are available to identify genomic alterations in individuals. First, sequencing allows for the detection of mutations. Sanger sequencing, the original method of sequencing, is an approach that allows analysis of only a few genes at the same time. NGS allows sequencing large number of DNA bases in a single run. This latter technology allows multigene sequencing for precision medicine purposes. In some specific platforms and conditions, NGS can also allow quantifying gene copy numbers, although CGH array or fluorescence in situ hybridization analyses are usually better for this purpose. Finally, gene expression array and reverse transcription polymerase chain reaction quantify gene expression. More recently, RNA sequencing is being developed to assess gene expression, translocations, and mutations.

\section{LEVEL OF EVIDENCE SCALE FOR DRUG TARGETS}

NGS and other high-throughput technologies analyze several hundred or thousands of genes in the same assay. These technologies can identify many genomic alterations in each patient. Nevertheless, only a few of them are truly implicated in the disease progression. A level of evidence scale that ranks

From the Department of Medical Oncology, Gustave Roussy, Villejuif, France.

Disclosures of potential conflicts of interest are found at the end of this article.

Corresponding author: Fabrice Andre, MD, Department of Medical Oncology, Gustave Roussy Institute, 114 Rue E Vaillant, Villejuif, France; email: fandre@igr.fr.

(c) 2015 by American Society of Clinical Oncology. 
targets according to their relevance has been developed to facilitate interpretation of these high-throughput assays, better communicate results to patients, and prioritize research needs. ${ }^{11}$ Level I evidence includes molecular alterations that have been shown to be validated targets, either based on phase III trials or several large phase I/II trials. Level II evidence includes molecular alterations associated with response to treatment in small or unique phase I/II trials. Level III molecular alterations include those that have been considered as promising targets based on preclinical studies. Finally, level IV evidence includes genomic alterations that are selected based on bioinformatics analyses only, without biologic studies to support them. This level of evidence scale also makes a difference according to whether the molecular alteration has been investigated in the same disease and whether studies included negative controls (evidence that patients without the alteration do not derive benefit from the targeted therapies). This level of evidence scale does not evaluate the medical usefulness of targeting the molecular alteration (benefit as compared with standard of care), but the antitumor activity obtained by targeting it. In the next sections of this article, we will use this level of evidence scale to classify molecular alterations in breast cancer. From this analysis, we will further discuss the current positioning of multiplex assay to treat patients with breast cancer.

\section{MOLECULAR TARGETS AND THEIR RESPECTIVE LEVEL OF EVIDENCE IN BREAST CANCERS}

Molecular alterations can be divided between drivers, mechanisms of resistance, mutational process and DNA-repair defects, immune alterations, cell death, angiogenesis, and metabolism. These latter three systems will not be discussed in the present article. The list of the most investigated molecular alterations and their level of evidence are reported in Table 1.

Oncogenic drivers can be defined as molecular alterations involved in malignant transformation and cancer progression. Targeting a driver is expected to lead to tumor shrinkage (known as oncogene deaddiction). As mentioned in the introduction, the two historic drivers include ER expression

\section{KEY POINTS}

Only a few molecular alterations are validated as targets in breast cancer (specifically ER and HER2 expression).

- Driver identification in breast cancers includes DNA-based analyses but also detection of pathway activation and dependency (e.g., ER, mTOR, and CDK4).

- Driver identification is not the sole application of genomics to personalize therapy for metastatic breast cancer.

- There is no evidence that using multiplex genomic testing for metastatic breast cancer improves outcomes.

- Ongoing trials are evaluating the medical usefulness of nextgeneration sequencing in metastatic breast cancers.

\section{TABLE 1. Potential Applications of Genomics for Metastatic Breast Cancers}

\begin{tabular}{|c|c|c|c|}
\hline $\begin{array}{l}\text { Application } \\
\text { of Genomics }\end{array}$ & $\begin{array}{l}\text { Optimal } \\
\text { Technology }\end{array}$ & Targets & $\begin{array}{l}\text { Level of Evidence } \\
\text { Associated with } \\
\text { the Target } 11\end{array}$ \\
\hline \multirow[t]{4}{*}{ Drivers (DNA) } & \multirow{4}{*}{$\begin{array}{l}\text { Next-generation } \\
\text { sequencing if } \\
\text { multiple genes } \\
\text { validated }\end{array}$} & $\begin{array}{l}\text { ERBB2 } \\
\text { amplification }\end{array}$ & I \\
\hline & & PIK3CA mutations & $\|$ \\
\hline & & AKT1 mutations & III \\
\hline & & ERBB2 mutations & III \\
\hline \multirow{3}{*}{$\begin{array}{l}\text { Drivers (RNA/ } \\
\text { proteins) }\end{array}$} & \multirow{3}{*}{$\begin{array}{l}\text { Gene expression } \\
\text { Phosphoprotein } \\
\text { assays }\end{array}$} & ER expression & 1 \\
\hline & & mTOR activation & ND \\
\hline & & CDK4/6 activation & ND \\
\hline $\begin{array}{l}\text { Lethal } \\
\text { subclone }\end{array}$ & $\begin{array}{l}\text { Ultra-deep } \\
\text { sequencing } \\
\text { Circulating DNA }\end{array}$ & ESR1 mutations & III \\
\hline DNA repair & $\begin{array}{l}\text { Targeted } \\
\text { sequencing } \\
\text { Whole-exome } \\
\text { sequencing } \\
\text { SNP arrays }\end{array}$ & BRCA1/2 mutations & $|/| I$ \\
\hline \multirow[t]{2}{*}{$\begin{array}{l}\text { Immune } \\
\text { system }\end{array}$} & \multirow{2}{*}{$\begin{array}{l}\text { Whole-exome } \\
\text { sequencing } \\
\text { RNA sequencing }\end{array}$} & $\begin{array}{l}\text { PD-L1 } \\
\quad \text { overexpression }\end{array}$ & ND \\
\hline & & Neoantigen & \\
\hline
\end{tabular}

Abbreviations: ER, estrogen receptor; mTOR, mammalian target of rapamycin; ND, not determined; PD-L1, programmed death ligand 1.

and ERBB2-amplification. These two targets are associated with level I evidence. Additional candidate molecular alterations are being investigated in breast cancer. These alterations should be divided between DNA-based assays and pathway-based assays. At the DNA level, there are between 10 to 20 genomic alterations that are currently the targets of drug development.

PIK3CA mutations are observed in approximately 25\% of breast cancers, mainly those with ER or HER2 expression. ${ }^{12}$ PI3K activates AKT1 that subsequently activates mTOR. AKT1 also interact with pathways that do not relate with mTOR, including FOXO, BAD, and GSK3. Several drug families target $\mathrm{PI} 3 \mathrm{~K} / \mathrm{AKT} / \mathrm{mTOR}$ pathways, including mTOR inhibitors, AKT inhibitors, nonselective PI3K inhibitors, and alpha-selective PI3K inhibitors. In an ancillary study of the BOLERO2 trial, PIK3CA mutations were not predictive for the efficacy of mTOR separate inhibitors in ER-positive/ HER2-negative breast cancer. ${ }^{13}$ Nonselective PI3K inhibitors target most of the PI3K subunits and thus present a narrow therapeutic index. These drugs can therefore achieve modest PI3K inhibition. This could explain why nonselective PI3K inhibitors have shown mitigated results in phase II randomized trials. ${ }^{14}$ Conversely, alpha-selective inhibitors are very potent inhibitors of alpha subunit, a major player of PI3K activity in cancer. Interestingly, phase I studies using alphaselective PI3K inhibitors have shown extremely encouraging results in patients who with $P I K 3 C A$ mutations, suggesting that PIK3CA mutation could be a relevant target in mBC. ${ }^{15,16}$ This genomic alteration should be ranked level IIa. Activating AKT1 mutation is the other genomic alteration located in 
the pathway. First, $A K T 1$ mutations occur in approximately $3 \%$ of breast cancers. These alterations are oncogenic in preclinical models and have been associated with objective response to mTOR inhibitors in molecular screening programs. ${ }^{10}$ Ongoing studies are investigating the efficacy of AKT inhibitors in patients with this genomic alteration. Although some retrospective analyses suggest oncogene deaddiction in patients with $A K T 1$ mutations, this target is still considered to be a level III target.

FGFR1 amplifications occur in approximately $10 \%$ of breast cancers, mainly those with ER expression. This alteration has been associated with very promising results in preclinical studies. ${ }^{17}$ Nevertheless, treatment with highly specific and bioactive FGFR inhibitors failed to demonstrate antitumor activity in phase I trials. ${ }^{18}$ Interestingly, multikinase inhibitors like lucitanib were associated with promising antitumor activity in patients presenting FGFR1 amplifications, ${ }^{19}$ but whether this antitumor activity relates to FGFR inhibition is unclear. FGFR1 amplification could be associated with level III evidence as a target. CCND1 is amplified in approximately $15 \%$ of breast cancers. This amplification is not clearly associated with CCND1 expression, and clinical studies have failed to validate that CCND1 amplification are relevant targets in breast cancer. ${ }^{20}$ Finally, the last interesting target located on tyrosine kinase is ERBB2 mutations. These mutations have been shown to be activating mutations and to be associated with antitumor activity of neratinib, a HER2 inhibitor. ${ }^{21}$ Phase II trials are ongoing. This target is currently considered as level III evidence but could jump to level I if consistent studies report high levels of antitumor activity for neratinib in this genomic segment.

Besides alterations at the DNA levels, assessing pathway activation and dependency could provide relevant information about driving forces of cancer progression. To illustrate, ER expression drives cancer progression in ER-positive breast cancer, although no alteration is usually detected at the DNA level. This emphasizes the relevance of assessing pathway activation in breast cancer. The Cancer Genome Atlas data have suggested that PI3K/AKT/mTOR and CDK4/Rb pathways are the two most relevant targetable pathways in breast cancer. Gene expression signatures could provide information about activation status of these pathways, together with assessment of phosphoproteins. For example, in the BOLERO3 trial, biomarker studies showed that activation of mTOR (specifically PS6K) is associated with high sensitivity to everolimus.

Emergence of lethal subclone is a well-described mechanism of resistance to targeted therapies. In patients with lung cancer, it has been well documented that T790M mutations, although the minority at the time of diagnosis, become predominant after resistance to EGFR inhibitors. ${ }^{22}$ In breast cancer, a similar phenomenon is being observed with ESR1. ESR1 is the gene that encodes for ER. Although less than 1\% of early breast cancers present mutations, it is considered that between $10 \%$ to $30 \%$ of breast cancers resistant to aromatase inhibitors will have a hotspot mutation thus leading to ligand-independent activation of the receptor. ${ }^{23,24}$ It has been suggested from preclinical works that high-dose fulvestrant could present some antitumor activity in this subset of patients. Several new ER degraders like GDC-0810 are being developed in this setting (NCT01823835). Until now, this target is classified level III. There are several questions surrounding this genomic alteration that could have some clinical influence. First, as opposed to T790M, this mutation has not yet been reported in a minority subclone in a primary tumor. Finding the ESR1 mutation in a minority clone in a primary tumor would open the path for the development of ultra-deep sequencing to detect them and potentially treating them very early during the disease course. Second, the use of circulating DNA could help detect these mutations during the disease course and treat them early. Finally, one study has suggested that ESR1 mutations could be associated with very poor outcome. If validated, this finding would suggest that this genomic segment would deserve some fast-track approvals based on phase II data.

The third application for genomic tests is the identification of DNA repair defects and mutational processes at the individual level. Identifying DNA repair defects could lead to administration of personalized synthetic lethality strategies or specific genotoxic agents. The best example in breast cancer is provided by BRCA1 and BRCA2 mutations. When biallelic, BRCA1 and $B R C A 2$ mutations and/or loss lead to homologous recombination deficiency and genomic instability. BRCA1/2 mutations have been associated with sensitivity to PARP1 inhibitors (synthetic lethality) and DNA alkylating agents (genotoxic). ${ }^{25}$ Interestingly in these trials, patients without $B R C A 1 / 2$ alterations did not present a similar degree of antitumor activity as compared with patients with mutations. Based on these consistent data from phase III and large phase II programs, BRCA1/2 mutations as drug targets have a level I evidence. ${ }^{11}$ The controversy in this area is more about how to position each therapeutic strategy (PARP1 inhibition and DNA alkylating agents) and to show medical usefulness over standard of care, rather than whether BRCA1/2 mutations constitute a target per se. The second controversy is about whether some functional tests evaluating homologous recombination deficiency (HRD) could have better performance than detecting $B R C A 1 / 2$ mutations. HRD tests could have better performance either by selecting the right patient with the BRCA1/2 mutation or by identifying patients with BRCA1/2 wild-type who present with HRD. When assessed retrospectively, the HRD test developed by Myriad was not associated with a differential sensitivity between platinum and docetaxel. The HRD test developed by Clovis has been associated with sensitivity to the PARP inhibitor rucaparib, even in the absence of BRCA mutation. ${ }^{26}$ An ongoing phase II trial (RUBY) is testing whether rucaparib could present antitumor activity in patients with BRCA1/2 wild-type who present a high $\mathrm{HRD}$. Beyond HRD and BRCA, assessing other DNA repair genes or pathways could allow expanding the array of patients who could be eligible for synthetic lethality strategies. ATM and ATR mutations are observed in approximately $2 \%$ of breast cancers and could define a subset of patients eligible for synthetic lethality approaches. In terms of pathways, several studies have suggested that the mutational pattern detected by whole-exome sequencing could allow for defining which DNA repair pathway is 
altered in each individual. ${ }^{27}$ This could potentially lead to the use of whole-exome sequencing to individualize synthetic lethality approaches for patient treatment.

Finally, the fourth potential application field of genomics to individualize therapy is immunology. Genomics could allow detecting neoantigens and expression of ligands for immune checkpoints, ${ }^{28}$ but also test the competence of the cancer cell to present antigens and to be killed. Recent data obtained in metastatic triple-negative breast cancer suggest that anti-PD-1 antibody could present some antitumor activity. ${ }^{29}$ Finally, genomic tests could evaluate whether the host could generate an antitumor immune response following immunogenic cell death. ${ }^{30}$ For example, TLR4 polymorphisms confer lack of immunogenicity and have been associated with resistance to anthracyclines in patients with breast cancer. ${ }^{30}$

\section{CLINICAL DEVELOPMENT OF PRECISION MEDICINE IN METASTATIC BREAST CANCER: WHICH TECHNOLOGY? WHICH SAMPLES? WHICH TRIALS?}

As discussed in the previous section, there are large numbers of applications for genomics to better treat patients in the metastatic setting. Each application deserves a specific technology and it is important to define which technology will be developed for which purpose. Current approaches of precision medicine aims at identifying drivers at the DNA level. For this purpose, sequencing is the best technology. Whether sequencing should be based on Sanger technology or should consist in NGS depends on the number of genes to be tested. An important aspect of breast cancer is the high number of copy number alterations that could potentially drive cancer progression. Assessing gene copy numbers requires fluorescence in situ hybridization technology for a single or a few genes, or CGH/SNP arrays for a large number of genes. Interestingly, some centers can now robustly assess copy number using NGS technology. ${ }^{9}$ This therefore makes this technology a preferred choice for clinical research programs that aim to identify drivers in $\mathrm{mBC}$. Alternatively, circulating DNA could be useful when biopsies are not feasible. As mentioned previously, assessment of pathways activation could be done by gene expression arrays, reverse transcription polymerase chain reaction, or phosphoprotein assays. Other technologies could be dedicated to specific purposes of clinical research. First, ultra-deep sequencing could be an interesting approach to detect minority clones, and circulating DNA could be an interesting approach to detect the appearance of resistance. SNP array could be interesting to quantify HRD. On a long-term perspective, one could argue that the best approach to personalize therapy will be to apply wholeexome sequencing to hard-to-treat $\mathrm{mBC}$. Whole-exome sequencing offers the advantage of detecting both drivers, DNA repair defects and neoantigens. ${ }^{28}$ Finally, RNA sequencing could offer the advantage of detecting pathway activation and target expression.

One field for controversy is which sample should be used for target identification in patients. Primary tumors offer the advantage of being accessible and not requiring additional biop- sies. Nevertheless, several studies have shown that targets can be lost or gained during the disease evolution. ${ }^{31}$ More recently, evidence has been reported that targets acquired during the disease course-although not trunk alterations-could drive cancer progression. ${ }^{32}$ This emphasizes the need to assess genomic and molecular targets at the time of treatment decision and start. Assessing cancer biology at the time of treatment decision would need to perform biopsies of metastatic sites. Whenever this is feasible, biopsy of metastatic sites should be the priority since it allows assessing genomic alterations but also RNA and protein expression together with immune markers. Nevertheless, if the tumor site is difficult to biopsy or in case of bone disease, circulating DNA could be a possible alternative.

Until now, there has been no data to support the use of DNA- or RNA-based technologies in daily practice for patients with $\mathrm{mBC}$. Several nonrandomized trials have been performed but they did not provide a clear picture about the potential medical usefulness of precision medicine for breast cancer. In the SAFIR01 trial, ${ }^{10}$ approximately $28 \%$ of the patients presented evidence of antitumor activity but only $10 \%$ had an objective response. These numbers align with phase I/II trials that tested drugs without molecular selection. In a clinical trial testing gene expression, Von Hoff et al reported that a group of patients had their progressive-free survival prolonged under genomic-based therapy. ${ }^{33}$ Nevertheless, this trial did not have a control group with patients treated with the same treatments but not driven by genomics. Outside of these two trials, there is no large study reporting efficacy of genomics in $\mathrm{mBC}$, and therefore, with the exception of prospective clinical trials, multiplex approaches cannot be recommended for routine practice.

The next question is how to provide evidence that the use of genomics improves outcomes in patients with $\mathrm{mBC}$. There are two possible strategies to address this question. The first strategy consists of prospectively validating each target in large clinical trials. This strategy is currently being used in most of the clinical programs in breast cancer. For example, HER2 inhibitors are being developed in patients with ERBB2 mutations, PI3K inhibitors are being developed in patients with PIK3CA mutations, AKT inhibitors in patients with AKT1 mutations, and so on. This approach will lead to the clinical validation of several genomic alterations. Once the number of such genomic alterations is large enough, companion diagnosis will very likely switch from Sanger single-gene sequencing to NGS. The second approach will consist of the clinical validation of a multiplex approach. In this design, the trial does not aim to validate each genomic alteration, but to test the hypothesis that using a multiplex technology improves outcomes. For example, the SAFIR02 trial (NCT02299999) is testing the hypothesis that use of NGS and CGH arrays improves outcomes compared with standard of care.

\section{MOVING TO RATIONALE COMBINATIONS}

As previously mentioned, breast cancer is a complex disease in which each patient could present several altered genomic alterations and pathways. Therefore, there is a strong rationale to 
combine drugs in the era of molecular medicine. There are three possible ways of combining drugs. First, drugs can be combined based on the presence of multiple genomic alterations. It has been suggested that multiple genomic alterations on drivers could be associated with resistance to therapy. ${ }^{34}$ Combining drugs that target different genomic alterations could therefore lead to antitumor activity. This is the rationale to combine HER2 and PI3K inhibitors in patients with ERBB2-amplified, PIK3CA-mutated BC. The second rationale to combine therapies would be to target molecular processes in different systems. For example, ERBB2-amplified $\mathrm{BC}$ is associated with a high level of PD-L1 expression and PD-L1-induced immune suppression. There is therefore a rationale to combine HER2-inhibitors and anti-PD-1 in patients presenting ERBB2-amplified and PD-L1induced immune suppression (PANACEA; NCT02129556). Finally, other combinations will aim at avoiding cancer cell adaptation. For example, cancer cells presenting a PIK3CA mutation can further adapt to PI3K inhibitors through CDK4 or mTOR activation. This provided a rationale to evaluate triple combinations therapy.

\section{CONCLUSIONS AND PERSPECTIVES}

A high number of gene or molecular alterations could be considered as actionable in breast cancer. Nevertheless, only a few of them (ER, HER2 expressions, and-to a lesser extent-BRCA and PIK3CA mutations) are currently validated as relevant targets. There is therefore no robust evidence that using a multiplex technology in mBC improves patient outcomes. Nevertheless, this approach could allow for accelerated drug development by detecting patients with genomic alterations and driving them to phase I/II trials. The current approach to validating genomics for $\mathrm{mBC}$ consists of testing drug efficacy in each genomic segment by validating each molecular alteration one by one. Since most of these genomic alterations are rare, there is a need to develop very large molecular screening programs. Several international screening programs have been set up recently including the AURORA program, developed by Breast International Group. Alternative development of precision medicine could consist of evaluating the overall effect of sequencing technologies in the whole population of patients with breast cancer, independently of each single alteration. This approach is being used in the SAFIR02 trial. Finally, one of the major challenges in the future will be optimally to implement these new technologies to secure access to innovations for all patients. To achieve this goal, the Institut National du Cancer has set up 28 public genomic centers in France that have a goal to offer access to genomic tests for free to all patients with cancer. This model, initially based on Sanger sequencing, is currently moving to NGS.

\section{Disclosures of Potential Conflicts of Interest}

Relationships are considered self-held and compensated unless otherwise noted. Relationships marked "L" indicate leadership positions. Relationships marked "I" are those held by an immediate family member; those marked "B" are held by the author and an immediate family member. Institutional relationships are marked "Inst." Relationships marked "U" are uncompensated.

Employment: None. Leadership Position: None. Stock or Other Ownership Interests: None. Honoraria: Fabrice Andre, AstraZeneca, Novartis. Consulting or Advisory Role: Fabrice Andre, Astellas Pharma, AstraZeneca, Novartis. Speakers' Bureau: None. Research Funding: Fabrice Andre, AstraZeneca (Inst), Novartis (Inst), Roche/Genentech (Inst). Patents, Royalties, or Other Intellectual Property: None. Expert Testimony: None. Travel, Accommodations, Expenses: Fabrice Andre, GSK, Novartis, Roche. Other Relationships: None.

\section{References}

1. Early Breast Cancer Trialists' Collaborative Group (EBCTCG). Effects of chemotherapy and hormonal therapy for early breast cancer on recurrence and 15-year survival: an overviewof the randomised trials. Lancet. 2005;365:1687-1717.

2. Vogel CL, Cobleigh MA, Tripathy D, et al. Efficacy and safety of trastuzumab as a single agent in first-line treatment of HER2-overexpressing metastatic breast cancer. J Clin Oncol. 2002;20:719-726.

3. Perou CM, Sørlie T, Eisen MB, et al. Molecular portraits of human breast tumours. Nature. 2000;406:747-752.

4. Sørlie T, Perou CM, Tibshirani R, et al. Gene expression patterns of breast carcinomas distinguish tumor subclasses with clinical implications. Proc Natl Acad Sci U S A. 2001;98:10869-10874.

5. Sotiriou C, Neo SY, McShane LM, et al. Breast cancer classification and prognosis based on gene expression profiles from a population-based study. Proc Natl Acad Sci U S A. 2003;100:10393-10398.

6. Lehmann $\mathrm{BD}$, Bauer JA, Chen X, et al. Identification of human triple-negative breast cancer subtypes and preclinical models for selection of targeted therapies. J Clin Invest. 2011;121:27502767.

7. Stephens PJ, Tarpey PS, Davies H, et al. The landscape of cancer genes and mutational processes in breast cancer. Nature. 2012;486: 400-404.

8. Cancer Genome Atlas Network. Comprehensive molecular portraits of human breast tumours. Nature. 2012;490:61-70.

9. Eisenstein M. Foundation medicine. Nat Biotechnol. 2012;30:14.

10. André F, Bachelot T, Commo F, et al. Comparative genomic hybridisation array and DNA sequencing to direct treatment of metastatic breast cancer: a multicentre, prospective trial (SAFIR01/UNICANCER). Lancet Oncol. 2014;15:267-274.

11. Andre F, Mardis E, Salm M, et al. Prioritizing targets for precision cancer medicine. Ann Oncol. 2014;25:2295-2303.

12. Stemke-Hale K, Gonzalez-Angulo AM, Lluch A, et al. An integrative genomic and proteomic analysis of PIK3CA, PTEN, and AKT mutations in breast cancer. Cancer Res. 2008;68:6084-6091. 
13. Hortobagyi GN, Piccart-Gebhart MJ, Rugo HS, et al. Correlation of molecular alterations with efficacy of everolimus in hormone receptor-positive, HER2-negative advanced breast cancer: Results from BOLERO-2. J Clin Oncol. 2013;31 (suppl; abstr LBA509).

14. Krop I, Johnston S, Mayer IA, et al. The FERGI phase II study of the PI3K inhibitor pictilisib (GDC-0941) plus fulvestrant vs fulvestrant plus placebo in patients with $\mathrm{ER}+$, aromatase inhibitor (AI)-resistant advanced or metastatic breast cancer-Part I results. Cancer Res. 2014;74 (suppl; abstr S2-02).

15. Saura C, Sachdev J, Patel MR, et al. Ph1b study of the PI3K inhibitor taselisib (GDC-0032) in combination with letrozole in patients with hormone receptor-positive advanced breast cancer. Cancer Res. 2014; 74 (suppl; abstr PD5-2).

16. Shah PD, Moynahan ME, Modi S, et al. Phase I trial: PI3Ka inhibitor BYL719 plus aromatase inhibitor (AI) for patients with hormone receptor-positive $(\mathrm{HR}+)$ metastatic breast cancer $(\mathrm{MBC})$. Cancer Res. 2014;74 (suppl; abstr PD5-3).

17. Turner N, Pearson A, Sharpe R, et al. FGFR1 amplification drives endocrine therapy resistance and is a therapeutic target in breast cancer. Cancer Res. 2010;70:2085-2094.

18. Andre F, Ranson M, Dean E, et al. Results of a phase I study of AZD4547, an inhibitor of fibroblast growth factor receptor (FGFR), in patients with advanced solid tumors. Cancer Res. 2013;73 (suppl; abstr LB-145).

19. Soria JC, DeBraud F, Bahleda R, et al. Phase I/IIa study evaluating the safety, efficacy, pharmacokinetics, and pharmacodynamics of lucitanib in advanced solid tumors. Ann Oncol. 2014;25:2244-2251.

20. Finn RS, Crown JP, Lang I, et al. The cyclin-dependent kinase $4 / 6$ inhibitor palbociclib in combination with letrozole versus letrozole alone as first-line treatment of oestrogen receptor-positive, HER2-negative, advanced breast cancer (PALOMA-1/TRIO-18): a randomised phase 2 study. Lancet Oncol. 2015;16:25-35.

21. Bose R, Kavuri SM, Searleman AC, et al. Activating HER2 mutations in HER2 gene amplification negative breast cancer. Cancer Discov. 2013;3: 224-237.

22. Lee Y, Lee GK, Lee YS, et al. Clinical outcome according to the level of preexisting epidermal growth factor receptor T790M mutation in patients with lung cancer harboring sensitive epidermal growth factor receptor mutations. Cancer. 2014;120:2090-2098.
23. Robinson $\mathrm{DR}, \mathrm{Wu} \mathrm{YM}$, Vats $\mathrm{P}$, et al. Activating ESR1 mutations in hormone-resistant metastatic breast cancer. Nat Genet. 2013;45:1446-1451.

24. Toy W, Shen $\mathrm{Y}$, Won $\mathrm{H}$, et al. ESR1 ligand-binding domain mutations in hormone-resistant breast cancer. Nat Genet. 2013;45:1439-1445.

25. Tutt A, Robson M, Garber JE, et al. Oral poly(ADP-ribose) polymerase inhibitor olaparib in patients with BRCA1 or BRCA2 mutations and advanced breast cancer: a proof-of-concept trial. Lancet. 2010;376:235-244.

26. Swisher E, Brenton J, Kaufmann S, et al. Updated clinical and preliminary correlative results of ARIEL2, a Phase 2 study to identify ovarian cancer patients likely to respond to rucaparib. Eur J Cancer. 2014;50: suppl 6, Abstr 215.

27. Alexandrov LB, Nik-Zainal S, Wedge DC, et al. Signatures of mutational processes in human cancer. Nature. 2013;500:415-421.

28. Snyder A, Makarov V, Merghoub T, et al. Genetic basis for clinical response to CTLA-4 blockade in melanoma. NEngl J Med. 2014;371:21892199.

29. Nanda R, Chow LQ, Dees EC, et al. A phase Ib study of pembrolizumab (MK-3475) in patients with advanced triple-negative breast cancer. Cancer Res. 2014;74 (suppl; abstr S01-09).

30. Apetoh L, Ghiringhelli F, Tesniere A, et al. Toll-like receptor 4-dependent contribution of the immune system to anticancer chemotherapy and radiotherapy. Nat Med. 2007;13:1050-1059.

31. Criscitiello C, André F, Thompson AM, et al. Biopsy confirmation of metastatic sites in breast cancer patients: clinical impact and future perspectives. Breast Cancer Res. 2014;16:205.

32. Favero F, McGranahan N, Salm M, et al. Glioblastoma adaptation traced through decline of an IDH1 clonal driver and macroevolution of a double minute chromosome. Annals of Oncol. In press.

33. Von Hoff DD, Stephenson JJ Jr, Rosen P, et al. Pilot study using molecular profiling of patients' tumors to find potential targets and select treatments for their refractory cancers. J Clin Oncol. 2010;28: 4877-4883.

34. Yu F, Ferté C, Soria J, et al. Combination of targetable gene alterations decreases anti-cancer drug treatment response in cell lines and patients. Paper presented at: Cancer Pharmacogenomics and Targeted Therapies 2014; 17-19 September 2014; Welcome Trust Genome Campus, Hinxton, Cambridge, United Kingdom. 